Jurnal Keperawatan Padjadjaran

ISSN 2338-5324 (print)

ISSN 2442-7276 (online)

Online di http://jkp.fkep.unpad.ac.id

DOI : $10.24198 / \mathrm{jkp}$

\title{
Analysis of Factor Affecting Nutrition Status on Children
}

\author{
Laili Rahayuwati ${ }^{1}$, Ikeu Nurhidayah ${ }^{1}$, Nur Oktavia Hidayati' ${ }^{1}$, Sri Hendrawati', \\ Habsyah Saparidah Agustina ${ }^{1}$, Rindang Ekawati ${ }^{2}$ \\ ${ }^{1}$ Faculty of Nursing Universitas Padjadjaran, Bandung, Indonesia \\ ${ }^{2}$ Research and Development, National Indonesian Population Board, Bandung, Indonesia \\ Corresponding Email: laili.rahayuwati@unpad.ac.id
}

Submitted: 18-02-2019 Accepted: 12-06-2019 Published: 01-08-2019

\begin{abstract}
The problem of malnutrition remains overlooked in Indonesia, especially on children, caused by various factors. Indonesia is the $17^{\text {th }}$ country with 3 nutrition problems, including stunting (short body), wasting (skinny body), and overweight (obesity). This research aims to analyze factors affecting nutrition status on children in the area of West Java Province, including the mother's and the child's socio-demographics factor, and the child's health status. The research method was descriptive quantitative with cross-sectional approach. The number of samples is 810 . The research was conducted in 6 districts that support Family Planning (KB), including Bandung District, Bandung City, West Bandung District, Subang District, Sumedang District, and Garut District. The quantitative analysis consisted of univariates using percentage and frequency distribution, as well as bivariate analysis using chi square test. The result of the research shows that nearly all toddlers have good nutrition status as much as $87.9 \%$, and toddlers with malnutrition as much as $10.6 \%$. The analysis factor shows that there is a relationship between the mother's age $(p=0.048 ;$ OR $=1.583)$, family income $(p=0.010 ;$ OR $=1.803)$, delivery complications $(\mathrm{p}=0.008 ; \mathrm{OR}=2.091)$, provision of exclusive breastfed milk (ASI) at the age of $0-6$ years old $(\mathrm{p}=0.000$; OR $=2.321)$, provision of exclusive breast milk and complementary feeding given to babies before 6 months old (MPASI) at the age of 6 months to 2 years old $(\mathrm{p}=0.002 ; \mathrm{OR}=2.037)$, and the child's history of hospitalization $(\mathrm{p}=0.008$; $\mathrm{OR}=2.055)$, while other factors are considered irrelevant. This research suggests that healthcare staff collaborate in providing knowledge to mothers on the provision of exclusive breast milk and complementary feeding as well as the prevention of illness on their children.
\end{abstract}

Keywords: Children, factor affecting nutrition status, nutrition status. 
Laili Rahayuwati: Analysis of factor Affecting Nutrition Status on Children

\section{Introduction}

The problem of low-quality citizenship is indicated by, out of many others, the prevalence of nutrition affecting the quality of human resources. This will lead to the loss of young generations and the nation's future economy (Fotso et al., 2012). One of WHO's SDG targets is preventing hunger. In 2025, such problems of malnutrition are expected to be solved.

Indonesia is one of the developing countries with a quite serious malnutrition problem. Few others also face similar problems due to improper food provision (Ningsih, Kristiawati, \& Krisnana, 2014). Data from WHO (2010) discovered that 1.5 million children have died due to improper food provision and $90 \%$ of the cases occur in developing countries. Today, malnutrition remains a national problem unattended, especially on toddlers. This is because toddlers have a relatively fast growth, thus requiring the biggest portion of nutrition compared to other stages of development (Ningsih, Kristiawati, \& Krisnana, 2014).

Global Nutrition Report (USAID, 2014) claimed that Indonesia is the 17th country with 3 main malnutrition problems to date, including stunting (short body), wasting (skinny body), and overweight (obesity). Data from Basic Health Research (Riskesdas) (2013) show that the prevalence of fat body at the age of 0-59 months, according to weight/ height, is $11.8 \%$. Meanwhile, data from Nutrition Status Monitoring (Pemantauan Status Gizi (PSG)) claim that the prevalence of fat toddlers in Indonesia with similar measurements reaches 5.3\% (Kementerian Kesehatan Republik Indonesia, 2016). Data from Riskesdas (2013) also discovered that today's Indonesian children are suffering from malnutrition, knowing that 8 out of 100 children are obese. This prevalence is based on the index measurement of body mass divided by age on children aged 5-12 years old as much as $8 \%$. WHO (2010) thinks that malnutrition is not a problem in a country if that country has a fat-toddler indicator above $5 \%$.

Problems of nutrition are pertinent to a child lacking of nutrition at early age, impacting to his cognitive disabilities and behavior deceleration. A child's cognitive growth may also be affected by the resultant interaction between his brain and his environment. It also affects his intelligence, analytical thinking, and productivity level. Lack of nutrition in this age may be relevant to the risk of chronic disease such as obesity, heart and vein disease, hypertension, and diabetes. Parents, therefore, are responsible for providing adequate nutrition intake to support their child's cognitive development (Ministry of Health (Kemenkes RI), 2017; Mohd Nasir et al., 2012). It is one of the fundamental factors affecting the cognitive performance as a defense mechanism to nerve building and brain development (Kementerian Kesehatan Republik Indonesia, 2017; Mohd Nasir et al., 2012).

The nutrition status during the 1000 first days of birth (HPK), or also known as "heydays", will impact on the qualities of a child's health, intellect, and productivity in the future (USAID, 2014; Kementerian Kesehatan Republik Indonesia, 2013). Mothers and babies must have adequate and quality nutrition to ensure their own health and motoric, social, and cognitive abilities, as well as learning skills in the future. Children with malnutrition during their heydays will likely to face neurological issues, a decrease in learning abilities, a higher chance of dropping out from school, barriers in productivity and working motivation, a decrease in the ability of providing nutritious food, and a decrease in caretaking. These all will further result in the prevalence of malnutrition and poverty for more generations to come. Considering the importance of nutrition during heydays, prevention of nutrition problems, therefore, becomes the main priority to improve the life quality of future generations (Bappenas RI, 2012).

The efforts of prevention of nutrition problems must be adjusted with the collateral factors (Aridiyah, Rohmawati, \& Ririanty, 2015). The causing factor is directly from the child's unbalanced eating pattern and contagious illness. On the other hand, indirect factors include food availability, environment sanitation, and upbringing such as eating pattern, provision of knowledge, behavior, skills, and healthcare service (Arifin, 2016; Kementerian Kesehatan Republik Indonesia, 
2017; Subarkah, Nursalam, \& Rachmawati, 2016). This research aims to analyze factors that affect a child's nutrition status as seen by the mother's and the child's sociodemography, and the child's health status.

\section{Method}

This research is a descriptive quantitative research utilizing a cross-sectional approach. The population in this research is Productive Couples (PUS) with such criteria as pregnant mother (first to third trimester) and/or mothers with toddlers. These samples were taken from 6 districts in the province of West Java. The determination of districts for sampling in this research is done based on the highest number of occurrence of malnutrition in West Java (Bappenas RI, 2012). The districts include Bandung District, Bandung City, Garut District, West Bandung District, Subang District, and Sumedang District with the total of population as much as 534,652 respondents. The minimal number of samples is based on the number of population according to the table of sample determining by Isaac and Michael with 1\% significance rate and the total of population as many as $\pm 550,000$. Therefore, the minimal number of samples is $665-810$. The number of samples used in this research is 810 respondents. Data collection is carried out by enumerators by visiting respondents to each district. Filling in the questionnaire by the respondent was accompanied by enumerators.

Sampling technique is done using cluster sampling from 6 district consisting of 6 villages that support KB; therefore, each village is represented by 135 respondents in each. In this research, 810 respondents from $6 \mathrm{~KB}$ villages in 6 districts in West Java. Out of 810 respondents, 735 of which are toddlers and mother with toddlers $(89.3 \%)$, pregnant mother $(8.2 \%)$ or 67 people, and pregnant woman with toddlers $(2.5 \%)$ or approximately 20 people.

Data were collected by using questionnaire to measure: individual factors, consisting of the mother's history of pregnancy and medical records; socio-demographic factors, consisting of gender, age, education level, occupation, family income, health insurance, healthcare access, and behavior, including the family's knowledge and action in its sustainability, the effort of nutrition fulfillment on pregnant mother, antenatal check-up and secure delivery, exclusive breast milk provision, effort of nutrition fulfillment on toddlers, immunization provision, growth and development, and healthy life behavior on families (PHBS). Besides, sampling was also done by running through medical records taken from the Book of Mother and Child's Health to observe the health status of the mother and the child (nutrition problems, pregnant mother's anemia, a highrisk pregnancy), physical observation with anthropometry to observe nutrition status on toddlers classified as adequate nutrition, lack of nutrition, malnutrition, or over nutrition.

Data analysis was done using Chi Square and Spearman analysis to observe factors affecting nutrition status on children. Research relevant to humans must be done according to ethical principles. According to Polit and Beck (2008), researcher must provide protection toward individual rights involved in the nurse's research. Therefore, this research has guaranteed ethical consentment from research Ethical Committee Universitas Padjadjaran, with letter number: 1206/UN6/ $\mathrm{KEP} / \mathrm{EC} / 2018$. The implementation of this research was done using 3 months from October to December 2018.

Table 1 Nutrition Status of Toddlers based on Weight/Age

\begin{tabular}{lcc}
\hline \multicolumn{1}{c}{ Nutrition Status } & Frequency (f) & Percentage (\%) \\
\hline Malnutrition & 6 & 0.8 \\
Lack of Nutrition & 72 & 9.8 \\
Adequate Nutrition & 646 & 87.9 \\
Over Nutrition & 11 & 1.5 \\
\hline
\end{tabular}


Laili Rahayuwati: Analysis of factor Affecting Nutrition Status on Children

Table 2 Nutrition Status on Toddlers based on Distribution per District

\begin{tabular}{|c|c|c|c|c|c|c|c|c|c|c|c|c|}
\hline \multirow[t]{2}{*}{$\begin{array}{l}\text { Nutrition Status } \\
\text { Indicator }\end{array}$} & \multicolumn{2}{|c|}{$\begin{array}{c}\text { Bandung } \\
\text { City }\end{array}$} & \multicolumn{2}{|c|}{$\begin{array}{l}\text { Bandung } \\
\text { District }\end{array}$} & \multicolumn{2}{|c|}{$\begin{array}{c}\text { West } \\
\text { Bandung } \\
\text { District }\end{array}$} & \multicolumn{2}{|c|}{$\begin{array}{l}\text { Subang } \\
\text { District }\end{array}$} & \multicolumn{2}{|c|}{$\begin{array}{c}\text { Sumedang } \\
\text { District }\end{array}$} & \multicolumn{2}{|c|}{$\begin{array}{c}\text { Garut } \\
\text { District }\end{array}$} \\
\hline & f & $\%$ & f & $\%$ & $\mathbf{f}$ & $\%$ & f & $\%$ & f & $\%$ & f & $\%$ \\
\hline \multicolumn{13}{|l|}{ Based on Weight/Age: } \\
\hline Malnutrition & 2 & 1.6 & 0 & 0 & 0 & 0 & 1 & 0.8 & 3 & 2.6 & 0 & 0 \\
\hline Lack of Nutrition & 11 & 8.5 & 22 & 17.1 & 7 & 5.7 & 19 & 15.3 & 8 & 7 & 5 & 4.3 \\
\hline Adequate Nutrition & 114 & 89.1 & 106 & 82.1 & 116 & 94.3 & 99 & 79.9 & 102 & 88.7 & 109 & 94 \\
\hline Over Nutrition & 1 & 0.8 & 1 & 0.8 & 0 & 0 & 5 & 4 & 2 & 1.7 & 2 & 1.7 \\
\hline
\end{tabular}

Table 3 Mother's Socio-Demographic Factor

\begin{tabular}{|c|c|c|}
\hline Factors & Frequency (f) & Percentage $(\%)$ \\
\hline \multicolumn{3}{|l|}{ The mother's age } \\
\hline Early teens $12-16$ years old & 276 & 34.5 \\
\hline Late teens $17-25$ years old & 378 & 47.2 \\
\hline Early adults $26-35$ years old & 141 & 17.6 \\
\hline Late adults $36-45$ years old & 6 & 0.7 \\
\hline \multicolumn{3}{|l|}{ The mother's education level } \\
\hline Elementary school & 236 & 29.5 \\
\hline Middle school & 328 & 40.9 \\
\hline High school & 200 & 25.1 \\
\hline Diploma & 10 & 1.2 \\
\hline Undergraduate & 26 & 3.2 \\
\hline Postgraduate & 1 & 0.1 \\
\hline \multicolumn{3}{|l|}{ Mother's Occupation } \\
\hline Unemployed & 647 & 80.8 \\
\hline Farmer & 62 & 7.7 \\
\hline Private Employee & 48 & 6.0 \\
\hline Self-Employed & 31 & 3.9 \\
\hline Civil Servant & 3 & 0.4 \\
\hline Others & 10 & 1.2 \\
\hline \multicolumn{3}{|l|}{ Father's Occupation } \\
\hline Unemployed & 10 & 1.2 \\
\hline Farmer & 90 & 11.2 \\
\hline Private Employee & 208 & 26.0 \\
\hline Self-Employed & 396 & 49.5 \\
\hline Civil Servant & 40 & 5.0 \\
\hline Others & 57 & 7.1 \\
\hline \multicolumn{3}{|l|}{ Family income } \\
\hline IDR $1,500,000$ & 365 & 45.6 \\
\hline IDR $1,500,000-2,500,000$ & 271 & 33.8 \\
\hline
\end{tabular}


Laili Rahayuwati: Analysis of factor Affecting Nutrition Status on Children

\begin{tabular}{|c|c|c|}
\hline IDR $2,500,000$ & 165 & 20.6 \\
\hline \multicolumn{3}{|l|}{ Family Spending } \\
\hline IDR $1,500,000$ & 315 & 39.3 \\
\hline IDR $1,500,000-2,500,000$ & 332 & 41.5 \\
\hline IDR 2,500,000 & 154 & 19.2 \\
\hline \multicolumn{3}{|l|}{ Health Insurance } \\
\hline Negative & 282 & 35.2 \\
\hline Positive & 519 & 64.8 \\
\hline \multicolumn{3}{|l|}{ Access to Health Service } \\
\hline Difficult & 59 & 7.4 \\
\hline Easy & 742 & 92.6 \\
\hline \multicolumn{3}{|l|}{ Caretaker } \\
\hline Mother & 604 & 75.4 \\
\hline Grandmother/Grandfather & 153 & 19.1 \\
\hline Sibling & 40 & 5.0 \\
\hline Creche & 3 & 0.4 \\
\hline Help & 1 & 0.1 \\
\hline \multicolumn{3}{|l|}{ KB Acceptor } \\
\hline No & 146 & 18.2 \\
\hline Yes & 655 & 81.8 \\
\hline \multicolumn{3}{|l|}{ Types of KB } \\
\hline Injection & 440 & 67.2 \\
\hline IUD & 68 & 10.4 \\
\hline Piil & 105 & 16.0 \\
\hline Condom & 5 & 0.8 \\
\hline Implant & 24 & 3.7 \\
\hline MOW & 12 & 1.8 \\
\hline Mikrogi & 1 & 0.1 \\
\hline \multicolumn{3}{|c|}{$\begin{array}{l}\text { KB Information from Healthcare } \\
\text { Staff }\end{array}$} \\
\hline Negative & 35 & 4.4 \\
\hline Positive & 766 & 95.6 \\
\hline \multicolumn{3}{|l|}{ Mother's Nutrition Status } \\
\hline Very Skinny & 25 & 3.1 \\
\hline Skinny & 61 & 7.6 \\
\hline Normal & 379 & 47.3 \\
\hline Overweight & 180 & 22.5 \\
\hline Obese & 156 & 19.5 \\
\hline \multicolumn{3}{|l|}{ Pregnancy Status } \\
\hline Not Pregnant & 715 & 89.3 \\
\hline Pregnant & 86 & 10.7 \\
\hline
\end{tabular}


Laili Rahayuwati: Analysis of factor Affecting Nutrition Status on Children

\begin{tabular}{|c|c|c|c|c|}
\hline Negative & & & 779 & 97.3 \\
\hline Positive & & & 22 & 2.7 \\
\hline $\begin{array}{l}\text { Mother's } \\
\text { Illnesses }\end{array}$ & History of & Chronic & & \\
\hline Negative & & & 768 & 95.9 \\
\hline Positive & & & 33 & 4.1 \\
\hline
\end{tabular}

Table 4 Relationship between Mother's Factor and Child's Nutrition Status

\begin{tabular}{|c|c|c|c|c|c|}
\hline & & \multicolumn{2}{|c|}{ Nutrition Status (Weight/Age) } & \multirow[t]{2}{*}{$\mathrm{p}$ value } & \multirow[t]{2}{*}{ OR } \\
\hline & & Malnutrition & Adequate & & \\
\hline \multirow[t]{4}{*}{ Education Level } & \multirow{2}{*}{$\begin{array}{l}\text { Middle school and } \\
\text { under }\end{array}$} & 61 & 452 & \multirow[t]{4}{*}{0.783} & \multirow[t]{4}{*}{0.935} \\
\hline & & $11.9 \%$ & $88.1 \%$ & & \\
\hline & \multirow{2}{*}{$\begin{array}{l}\text { High school and } \\
\text { higher }\end{array}$} & 28 & 194 & & \\
\hline & & $12.6 \%$ & $87.4 \%$ & & \\
\hline \multirow[t]{4}{*}{ Age } & \multirow[t]{2}{*}{$>34$ years old } & 36 & 53 & \multirow[t]{4}{*}{0.048} & \multirow[t]{4}{*}{1.583} \\
\hline & & $15.7 \%$ & $23.0 \%$ & & \\
\hline & \multirow[t]{2}{*}{$\leq 34$ years old } & 194 & 452 & & \\
\hline & & $38.4 \%$ & $89.5 \%$ & & \\
\hline \multirow[t]{4}{*}{ Number of Children } & \multirow[t]{2}{*}{$>2$ children } & 18 & 137 & \multirow[t]{4}{*}{0.831} & \multirow[t]{4}{*}{0.942} \\
\hline & & $11.6 \%$ & $88.4 \%$ & & \\
\hline & \multirow[t]{2}{*}{$\leq 2$ children } & 71 & 509 & & \\
\hline & & $12.2 \%$ & $87.8 \%$ & & \\
\hline \multirow[t]{4}{*}{ Occupation } & \multirow[t]{2}{*}{ Employed } & 18 & 129 & \multirow[t]{4}{*}{0.955} & \multirow[t]{4}{*}{1.016} \\
\hline & & $12.2 \%$ & $87.8 \%$ & & \\
\hline & \multirow[t]{2}{*}{ Unemployed } & 71 & 517 & & \\
\hline & & $12.1 \%$ & $87.9 \%$ & & \\
\hline \multirow[t]{4}{*}{ Income } & \multirow[t]{2}{*}{$\leq \mathrm{IDR} 1,500,000$} & 52 & 283 & \multirow[t]{4}{*}{0.010} & \multirow[t]{4}{*}{1.803} \\
\hline & & $15.5 \%$ & $84.5 \%$ & & \\
\hline & \multirow[t]{2}{*}{$>$ IDR $1,500,000$} & 37 & 363 & & \\
\hline & & $9.3 \%$ & $90.8 \%$ & & \\
\hline \multirow[t]{4}{*}{ Spending } & $\leq$ IDR $1,500,000$ & 40 & 241 & 0.166 & 1.372 \\
\hline & & $14.2 \%$ & $85.8 \%$ & & \\
\hline & $>$ IDR $1,500,000$ & 49 & 405 & & \\
\hline & & $10.8 \%$ & $89.2 \%$ & & \\
\hline Health Insurance & Negative & 34 & 227 & 0.572 & 1.141 \\
\hline Possession & & $13.0 \%$ & $87.0 \%$ & & \\
\hline & Positive & 55 & 419 & & \\
\hline & & $11.6 \%$ & $88.4 \%$ & & \\
\hline Access to & Difficult & 5 & 54 & 0.376 & 0.653 \\
\hline Healthcare Service & & $8.5 \%$ & $91.5 \%$ & & \\
\hline & Easy & 84 & 592 & & \\
\hline & & $12.4 \%$ & $87.6 \%$ & & \\
\hline
\end{tabular}


Laili Rahayuwati: Analysis of factor Affecting Nutrition Status on Children

\begin{tabular}{|c|c|c|c|c|c|}
\hline \multirow[t]{4}{*}{ KB Acceptors } & Negative & 14 & 79 & 0.353 & 1.340 \\
\hline & & $15.1 \%$ & $84.9 \%$ & & \\
\hline & Yes & 75 & 567 & & \\
\hline & & $11.7 \%$ & $88.3 \%$ & & \\
\hline \multirow{4}{*}{$\begin{array}{l}\text { Mother's Nutrition } \\
\text { Status }\end{array}$} & Not Normal & 42 & 349 & 0.227 & 0.760 \\
\hline & & $10.7 \%$ & $89.3 \%$ & & \\
\hline & Normal & 47 & 297 & & \\
\hline & & $13.7 \%$ & $86.3 \%$ & & \\
\hline \multirow{4}{*}{$\begin{array}{l}\text { Delivery } \\
\text { Complications }\end{array}$} & Positive & 21 & 83 & 0.008 & 2.091 \\
\hline & & $20.2 \%$ & $79.8 \%$ & & \\
\hline & Negative & 68 & 562 & & \\
\hline & & $10.8 \%$ & $89.2 \%$ & & \\
\hline \multirow{4}{*}{$\begin{array}{l}\text { Delivery } \\
\text { Complications }\end{array}$} & Non-Healthcare & 7 & 47 & 0.842 & 1.088 \\
\hline & Staff & $13.0 \%$ & $87.0 \%$ & & \\
\hline & Healthcare staff & 82 & 599 & & \\
\hline & & $12.0 \%$ & $88.0 \%$ & & \\
\hline \multirow[t]{4}{*}{ Delivery Process } & Special delivery & 10 & 69 & 0.874 & 1.059 \\
\hline & & $12.7 \%$ & $87.3 \%$ & & \\
\hline & Normal delivery & 79 & 577 & & \\
\hline & & $12.0 \%$ & $88.0 \%$ & & \\
\hline \multirow{4}{*}{$\begin{array}{l}\text { History of } \\
\text { Contagious Illnesses }\end{array}$} & Positive & 3 & 20 & 0.889 & 1.092 \\
\hline & & $13.0 \%$ & $87.0 \%$ & & \\
\hline & Negative & 86 & 629 & & \\
\hline & & $12.1 \%$ & $87.9 \%$ & & \\
\hline \multirow{4}{*}{$\begin{array}{l}\text { History of Chronic } \\
\text { Illnesses }\end{array}$} & Positive & 3 & 30 & 0.588 & 0.716 \\
\hline & & $9.1 \%$ & $90.9 \%$ & & \\
\hline & Negative & 86 & 616 & & \\
\hline & & $12.3 \%$ & $87.7 \%$ & & \\
\hline
\end{tabular}

Table 5 The Child's Demography and Health Status Factor

\begin{tabular}{lcc}
\hline \multicolumn{1}{c}{ Factor } & Frequency (f) & Percentage (\%) \\
\hline Gender & 386 & 52.5 \\
Male & 349 & 47.5 \\
Female & & \\
Age & 733 & 99.7 \\
Toddlers & 2 & 0.3 \\
Children & & \\
Immunization Completeness & 17 & 2.3 \\
Non-Immunized & 102 & 13.9 \\
Partial Immunization & 616 & 83.8 \\
Complete Immunization & & \\
Immunization Schedule & & \\
\hline
\end{tabular}


Laili Rahayuwati: Analysis of factor Affecting Nutrition Status on Children

\begin{tabular}{|c|c|c|}
\hline Not Immunized & 17 & 2.3 \\
\hline Misscheduled Immunization & 118 & 16.1 \\
\hline On-time Immunization & 600 & 81.6 \\
\hline \multicolumn{3}{|l|}{$\begin{array}{l}\text { Status of Exclusive Breast Milk } \\
0-6 \text { Months }\end{array}$} \\
\hline $\begin{array}{l}\text { Exclusive Breast Milk and } \\
\text { Complementary Feeding }\end{array}$ & 59 & 8.0 \\
\hline $\begin{array}{l}\text { Exclusive Breast Milk and Formula } \\
\text { Milk }\end{array}$ & 117 & 16.0 \\
\hline Exclusive Breast Milk & 555 & 75.5 \\
\hline Only Formula Milk & 4 & 0.5 \\
\hline \multicolumn{3}{|l|}{$\begin{array}{l}\text { Status of Exclusive Breast Milk } 6 \\
\text { Month-2 years old }\end{array}$} \\
\hline $\begin{array}{l}\text { Exclusive Breast Milk, Formula } \\
\text { Milk, Complementary Feeding } \\
\text { (No) }\end{array}$ & 150 & 20.4 \\
\hline $\begin{array}{l}\text { Formula Milk and Complementary } \\
\text { Feeding (No) }\end{array}$ & 61 & 8.3 \\
\hline $\begin{array}{l}\text { Exclusive Breast Milk and } \\
\text { Complementary Feeding (Yes) }\end{array}$ & 524 & 71.3 \\
\hline \multicolumn{3}{|l|}{$\begin{array}{l}\text { Age During Which Complementary } \\
\text { Feeding is Given }\end{array}$} \\
\hline 0 Month & 2 & 0.3 \\
\hline 1 Month & 3 & 0.4 \\
\hline 2 Month & 4 & 0.5 \\
\hline 3 Month & 8 & 1.1 \\
\hline 4 Month & 19 & 2.6 \\
\hline 5 Month & 12 & 1.6 \\
\hline 6 Month (suitable) & 643 & 87.6 \\
\hline 7 Month & 33 & 4.5 \\
\hline 8 Month & 8 & 1.1 \\
\hline 9 Month & 1 & 0.1 \\
\hline 10 Month & 1 & 0.1 \\
\hline 12 Month & 1 & 0.1 \\
\hline \multicolumn{3}{|l|}{ History of Hospitalization } \\
\hline Negative & 624 & 84.9 \\
\hline Positive & 111 & 15.1 \\
\hline \multicolumn{3}{|l|}{ Child's Medical Records } \\
\hline Negative & 649 & 88.4 \\
\hline TBC & 18 & 2.4 \\
\hline ISPA/Pneumonia & 45 & 6.1 \\
\hline Other Illnesses & 23 & 3.1 \\
\hline
\end{tabular}


Laili Rahayuwati: Analysis of factor Affecting Nutrition Status on Children

Table 6 Relationship between Child's Health Status and Child's Nutrition Status

\begin{tabular}{|c|c|c|c|c|c|}
\hline & & \multicolumn{2}{|c|}{ Nutrition Status (Weight/Age) } & \multirow[t]{2}{*}{$\mathrm{p}$ value } & \multirow[t]{2}{*}{ OR } \\
\hline & & Malnutrition & Adequate & & \\
\hline \multirow[t]{4}{*}{ Gender } & Male & 43 & 343 & 0.398 & 0.826 \\
\hline & & $11.1 \%$ & $88.9 \%$ & & \\
\hline & Female & 46 & 303 & & \\
\hline & & $13.2 \%$ & $86.8 \%$ & & \\
\hline \multirow{4}{*}{$\begin{array}{l}\text { Immunization } \\
\text { Completeness }\end{array}$} & Partial & 11 & 108 & 0.297 & 0.703 \\
\hline & & $9.2 \%$ & $90.8 \%$ & & \\
\hline & Complete & 78 & 538 & & \\
\hline & & $12.7 \%$ & $87.3 \%$ & & \\
\hline \multirow{4}{*}{$\begin{array}{l}\text { Immunization } \\
\text { Schedule }\end{array}$} & Miss scheduled & 15 & 119 & 0.720 & 0.898 \\
\hline & & $11.2 \%$ & $88.8 \%$ & & \\
\hline & On-time & 74 & 527 & & \\
\hline & & $12.3 \%$ & $87.7 \%$ & & \\
\hline \multirow{4}{*}{$\begin{array}{l}\text { Exclusive Breast } \\
\text { Milk 0 - } 6 \text { Month }\end{array}$} & Non Exclusive & 35 & 141 & 0.000 & 2.321 \\
\hline & Breast Milk & $19.9 \%$ & $80.1 \%$ & & \\
\hline & Exclusive Breast & 54 & 505 & & \\
\hline & Milk & $9.7 \%$ & $90.3 \%$ & & \\
\hline \multirow{4}{*}{$\begin{array}{l}\text { Exclusive } \\
\text { Breast Milk and } \\
\text { Complementary } \\
\text { Feeding } 6 \text { Month - } \\
2 \text { years old }\end{array}$} & Negative & 38 & 173 & 0.002 & 2.037 \\
\hline & & $18.0 \%$ & $82.0 \%$ & & \\
\hline & Positive & 51 & 473 & & \\
\hline & & $9.7 \%$ & $90.3 \%$ & & \\
\hline \multirow{4}{*}{$\begin{array}{l}\text { Start of } \\
\text { Complementary } \\
\text { Feeding }\end{array}$} & Unsuitable & 14 & 78 & 0.330 & 1.359 \\
\hline & & $15.2 \%$ & $84.8 \%$ & & \\
\hline & Suitable & 75 & 568 & & \\
\hline & & $11.7 \%$ & $88.3 \%$ & & \\
\hline \multirow{4}{*}{$\begin{array}{l}\text { History of } \\
\text { Hospitalization }\end{array}$} & Positive & 22 & 89 & 0.008 & 2.055 \\
\hline & & $19.8 \%$ & $80.2 \%$ & & \\
\hline & Negative & 67 & 557 & & \\
\hline & & $10.7 \%$ & $89.3 \%$ & & \\
\hline \multirow[t]{4}{*}{ Medical Records } & Positive & 13 & 73 & 0.360 & 1.343 \\
\hline & & $15.1 \%$ & $84.9 \%$ & & \\
\hline & Negative & 76 & 573 & & \\
\hline & & $11.7 \%$ & $88.3 \%$ & & \\
\hline
\end{tabular}

Based on the table above, the nutrition status overall based on weight/age in 6 districts in nearly all cities are adequate $(87.99 \%)$, but malnourished children reach up to $10.6 \%$.
Seen from weight/age of the toddler's nutrition status (table 2) in 6 districts, nearly all of them have adequate nutrition, which is in Bandung City as much as $89.1 \%$, Bandung District, 82.1\%, Bandung Barat 
Laili Rahayuwati: Analysis of factor Affecting Nutrition Status on Children

District 94.3\%, Subang District 79.9\%, Sumedang District 88.7\%, and Garut District $94 \%$. However, Table 2 shows problems of malnourished toddlers. Out of 6 districts, 3 districs have malnourished and nutritionlacking children from more than $10 \%$, including Bandung City 10.1\%, Bandung District 17.1\%, and Subang District 16.1\%. On the other hand, the 3 remaining districts have the percentage below $10 \%$, including Sumedang District 9.6\%, Garut District 4.3\%, and West Bandung District 5.7\%. Bandung District and Subang District are the 2 areas with the biggest portion of malnutrition cases, as much as $17.1 \%$ and $15.3 \%$, respectively. The biggest number of malnutrition cases occurs in Sumedang District as much as $3.6 \%$, followed by Bandung City $2.6 \%$. Seen from above, Bandung District has the highest percentage for toddlers lacking of nutrition out of the 6 districts.

Overall, the prevalence of malnourished and nutrition-lacking nutrition in this research reaches up to $10.6 \%$. The distribution of the nutrition status for toddlers each district can be seen in this table 2 .

Table 3 above shows that the mother's age is highly varying, 47.2 mothers are aged $17-25$ years old, $40.9 \%$ others are junior high school graduates, $80.8 \%$ others are unemployed, thus they take care of their own children $75.4 \%$. The father's occupation is varying, but mostly are private employees $49.5 \%$ with average income lower than 1,500,000 rupiahs. The average family spending is not parallel with family income. As much as $41.5 \%$ of family spending is bigger than that of the income $(1,500,000$ rupiahs $2,500,000$ rupiahs). As much as $64.8 \%$ of all families have health insurance and $92.6 \%$ of which have easy access to healthcare service. As much as $81.8 \%$ of mothers have used family planning and $67.2 \%$ of mothers use injection $\mathrm{KB}$, while $9.6 \%$ get information about KB from healthcare staff. The mother's nutrition status is also varying, around $47.3 \%$ have adequate nutrition, and $3.1 \%$ lack of nutrition. As much as $89.3 \%$ of them are nonpregnant woman, $97.3 \%$ mothers do not have contagious illness history, and $95.9 \%$ mothers do not have history of chronic diseases.
Table 4 shows that the mother's sociodemographic factors are relevant to the nutrition status is the mother's age, family income, and delivery complications ( $p$ value $<0.05$ ), while other factors are considered irrelevant. Based on the OR value, it is found out that mothers above 35 years old carry the risk of having malnourished children 1.583 times bigger than those aged under 35 years old. In terms of family income, families having income less than 1,500,000 rupiahs carry the risk of having malnourished children 1,803 times bigger than those earning more than 1,500,000 rupiahs. On the other hand, mothers who have a history of delivery complications carry the risk of having malnourished children 2,091 times bigger than those who do not.

Table 5 shows that there are 386 male children (52.5\%). Nearly all children are toddlers $(99.7 \%)$. Based on Immunization status, nearly all children get complete Immunization $(83.8 \%)$, and nearly all get on-time Immunization (81.6\%). Besides, all children get exclusive breast milk $75.5 \%$ ). Most respondents get complementary feeding after the child is 6 month old $(71.3 \%)$ and nearly all respondents get complementary feeding after the child is 6 month old (87.6\%). On the other hand, based on the child's health status, nearly all children have never been hospitalised $(84.9 \%)$, and nearly all of them do not have any history of contagious illness $(88.4 \%)$.

The table above shows the relationship between the child's health factor and their nutrition status. Based on the $p$ value, it is known that the factor of exclusive breast milk provision on toddlers before six months old, exclusive breast milk and complementary feeding provision on toddlers aged 6 months - 2 years, and history of hospitalization with $\mathrm{p}$ value $<0.05$. This shows that there is a significant relationship between that variable and the child's nutrition status.

Based on the OR value, it was found out that a child not given exclusive breast milk only before 6 months old are at risk of being malnourished 2.321 times bigger than those who do. This also applies to child who do not get exclusive breast milk and 
complementary feeding, they are at risk of being malnourished 2.037 times bigger than those who do at the age of 6 months until 2 years old. On the other hand, the child with history of hospitalization have 2.055 risk bigger of having malnourished than those who do not.

\section{Discussion}

Overall, the toddler's nutrition status based on Weight/Age in 6 districts in most toddlers have adequate nutrition $(87.9 \%)$, but it can be seen that malnourished and nutrition-lacking toddlers reach $10.6 \%$. The nutrition status in 6 districts in nearly all children are categorized under "adequate," specifically in Bandung City 89.1\%, Bandung District 82.1, West Bandung District 94.3, Subang District 79.9, Sumedang District 88.7, and Garut District 94. Therefore, the problem of malnourished toddlers is still apparent. Out of these 6 locations, 3 districts have a more than $10 \%$ of malnourished and nutrition-lacking toddlers including Bandung City 10.1\%, Bandung District $17.1 \%$, and Subang District 16.1\%. Meanwhile, the remaining 3 districts have the percentage below 10\%, including Sumedang District 9.6\% and Garut District 4.3\%, West Bandung District 5.7\%. Bandung District and Subang District were the two areas with the biggest portion of malnourished toddlers; that is, $17.1 \%$ and $15.3 \%$ respectively. The biggest number of malnourished toddlers is in Sumedang District as much as 3.6\%, followed by Bandung City 2.6\%. Overall, the prevalence of malnourished and nutritionlacking toddlers reaches as high as $10.6 \%$. This number is generally lower than the national prevalence, which is $17.7 \%$ (Riskesdas, 2018), however this still exceed WHO parameter line in terms of the number of malnourished toddlers, which is $10 \%$.

Nutrition problems, especially malnourishment and stunting in toddlers, may the child's growth, with negative impacts lasting for future life, such as intellectual downturn, vulnerability of degenerative and non-contagious illnesses, productivity decrease, which then leads to poverty and the risk of having babies with low weight
(UNICEF, 2013; UNICEF, 2012; WHO, 2010; Adair \& Guilkey, 2007). Other impacts of malnutrition during heydays are always associated with lack of specific vitamin and minerals and other micronutrients. Past research regarding the impact of lack of micronutrients, from the increase of risk on contagious illness to death. The consequences for children is grave. Lack of pure protein in advanced stage may cause kwashiorkor during school age and adolescents.

The relationship between the mother's factor and the child's nutrition status can be seen from $p$ value on the table. If $p$ value shows $>0.05$, it means there is no relationship whatsoever. Otherwise, it shows a significant relationship. On the table, it was found out that the mother's factor relevant to the child's nutrition status is the mother's age, family income, and delivery complications. The results of this study are in line with Anugraheni and Kartasurya's research (2012) and Assefa, Belachew, and Negash's research (2013).

Based on the OR value, a mother aged above 35 years old has the risk of malnourished baby 1.583 times bigger than those aged below. This is parallel with research by Khotimah and Kuswandi (2014) claiming that age is an important indicator in determining one's productivity; younger people tend to have higher productivity level, while older ones tend to have lower. On the other hand, Harlock (2000) in Khotimah and Kuswandi (2014) claim that the older someone gets, the higher his level of knowledge is. According to Khotimah and Kuswandi (2014) and Astari, Nasoetion, and Dwiriani (2005) age is one of the factors that can describe how mature someone is, especially in terms of eating pattern, which will impact to the nutrition status. Therefore, younger mothers tend to create more varying eating pattern for their children, resulting in better nutrition status for both.

On the family income factor, it was known that families with income less than 1,500,000 rupiahs have the risk of malnourished toddlers 1803 times bigger than those who earn more. Limitation in income also determines the quality of food. 
Laili Rahayuwati: Analysis of factor Affecting Nutrition Status on Children

it is undeniable that what a family can earn will show in the food preserved in the dining table. Income is the main indicator relevant to food quality. The more a family can earn, the more is the quality of the food they consume. This argument sounds logical, knowing that a person can only eat what he can afford. Low income also leads to low purchasing power; this brings about many complications for the health of the family and the baby's nutrition status (Khotimah \& Kuswandi, 2014). The result of this research is also suitable with a literature claiming that poverty is the main cause of malnutrition, being the first of the list (Suhardjo, 2002). If seen from the characteristics of family income, the root of malnutrition problem lies in economic crises. Most toddlers having difficulty growing physically are born in low-economy family (Aridiyah, Rohmawati, \& Ririanty, 2015).

On the other hand, mothers who have delivery complications, have the risk of malnourished toddlers 2091 bigger than those who do not. These complications include anemia, hypertension, and hyperemesis. All will hamper the fetus's growth and development inside womb, so that the baby will be born with lower body weight (BBLR) which also impacts on his nutrition status (Karima \& Achadi, 2012).

Table 6 shows the relationship between the child's health and his nutrition status. Based on the $p$ value, the factor of exclusive breast milk provision on toddlers before the age of 6 months old, exclusive breast milk and complementary feeding provision for toddlers at the age of 6 months - 2 years old, and history of hospitalization have $p$ value $<0.05$. This shows that there is a significant relationship between that variable and the child's nutrition status. The results of this study are in line with Tan,s research (2011).

Based on the OR value, children who are not given exclusive breast milk only before 6 months old, carry the risk of malnutrition 2.321 times bigger than those who do. This is parallel with research from Nilakesuma, Jurnalis, and Rusjdi (2015) showing that toddlers getting exclusive exclusive breast milk have $80 \%$ normal nutrition status. This also applies to those who only get exclusive breast milk and complementary feeding carry the risk of malnutrition 2.037 times higher than those who get both at the age of 6 months to 2 years old.

The less frequency of exclusive breast milk provision becomes one of the triggers of malnutrition on toddlers, which may be caused by past experiences. A good exclusive breast milk provision by the mother will help balance the child's nutrition. This is because at the age of $0-6$ months, the mother will create an immunity system for the toddlers, preventing them from contagious illnesses. Afterwards. at the age of 6 months, the toddler is given complementary feeding in sufficient amount and frequency, so that their nutrition level is fulfilled. The lower the frequency of exclusive breast milk provision, the higher the risk of malnutrition to toddlers, seen from both weight/age and length/age indices.

A good provision of exclusive breast milk by mother will balance the child's nutrition so that his development goes normally. Exclusive breast milk is highly required during the baby's development period so that the nutrition is fulfilled. Therefore, it is mandatory that a mother provide exclusive breast milk to babies until 6 months and keep providing until the baby reaches 2 years old. (AL-Rahmad, Miko, \& Hadi, 2013). This research supports the Health Department claiming that issues during the baby's development is caused by lack of nutrition since birth, providing complementary feeding too early or too late, complementary feeding does not suffice the baby's needs or the providing pattern is less proper, as well as inadequate baby treatment. In this research, it can be concluded that there is a significant relationship between exclusive breast milk provision and the toddler's nutrition status $(p=0.000)$. This argument is strengthened by research from Giri, Suryani, and Murdani (2013) that mothers giving exclusive breast milk tend to have toddlers with better nutrition status than mothers who do not.

On the other hand, the child with history of hospitalization carries the risk 2.055 times bigger of malnourishment than children who do not. The status of lack of nutrition faced by toddlers is caused by the factor of history of contagious illness. This must be faced by the mother alone. This means that the history of contagious illness in toddlers is relevant to the fact that he has been infected 
on the respiratory system called ISPA; other illnesses include tuberculosis. All illnesses were faced by the toddlers themselves due to the amount of bacteria from food and nonhygienic environment. Such medical records allow the children to undergo hospitalization. Therefore, that very record is what hampers the nutrition status to be better (Handayani, 2017).

\section{Conclusion}

The research was done in 6 districts including Bandung City, Bandung District, West Bandung District, Subang District, Sumedang District, and Garut District. In general, the prevalence of toddlers with malnutrition reaches $10.6 \%$. This number is collectively lower than the national prevalence (17.7\%) (Riskesdas, 2018), but it exceeds the target from WHO (10.0\%). Therefore, this research concludes that there is a relationship between the mother's age $(\mathrm{p}=0.048$; OR $=1.583)$, family income $(\mathrm{p}=0.010 ; \mathrm{OR}=1.803)$, delivery complications $(\mathrm{p}=0.008$; OR $=$ $2.091)$, provision of exclusive breast milk at the age of $0-6$ years old $(\mathrm{p}=0.000$; OR $=2.321$ ), provision of exclusive breast milk and complementary feeding at the age of 6 month-2 years old $(\mathrm{p}=0.002 ; \mathrm{OR}=2.037)$, and the child's history of hospitalization ( $p$ $=0.008 ; \mathrm{OR}=2.055)$, while other factors are irrelevant. Mothers with the age above 35 , family with income less than 1,500,000 rupiahs, mothers with delivery complications, children not given exclusive breast milk before 6 months old, children not given exclusive breast milk and complementary feeding, and children with history of hospitalizationall these factors lead to them having bigger risks of malnutrition. Therefore, the research suggests that healthcare staff collaborate from all sectors to provide health education on the provision of exclusive breast milk and complementary feeding as well as on the prevention of illness on children.

\section{References}

Adair, L.S., \& Guilkey, D.K. (2007). Agespecific determinants of stunting in Filipino children. Journal of Nutrition, 127, 314-320.

AL-Rahmad, A.H., Miko, A., \& Hadi, A. (2013). Kajian stunting pada anak balita ditinjau dari pemberian ASI eksklusif, MP-ASI, status imunisasi dan karakteristik keluarga di Kota Banda Aceh, (Study of Stunting in Children Under Five in Terms of Exclusive Breastfeeding, Complementary Feeding, Immunization Status and Family Characteristics in Banda Aceh City). Jurnal Kesehatan Ilmiah Nasuwakes, 6(2), 169 184.

Anugraheni, H.S., \& Kartasurya, M.I. (2012). Faktor risiko kejadian stunting pada anak usia 12-36 bulan di Kecamatan Pati, Regency Pati, (Risk factors for the Incidence of Stunting in Children Aged 12-36 Months in Pati District). Eprints Undip.

Aridiyah, F.O., Rohmawati, R., \& Ririanty, M. (2015). Faktor-faktor yang mempengaruhi kejadian stunting pada anak balita di wilayah pedesaan dan perkotaan (The factors affecting stunting on toddlers in rural and urban areas). e-Jurnal Pustaka Kesehatan, 3(1), 163-170.

Arifin, Z. (2016). Gambaran pola makan anak usia 3-5 years old dengan gizi kurang di Pondok Bersalin Tri Sakti Balong Tani Kecamatan Jabon-Sidoarjo, (Overview of Diet for Children aged 3-5 years old with Malnutrition in Tri Sakti Balong Tani Maternity Hut, Jabon-Sidoarjo District). Midwiferia, 1(1), 16. https://doi. org/10.21070/mid.v1i1.345.

Assefa H., Belachew T., \& Negash L. (2013). Socioeconomic factors associated with underweight and stunting among adolescents of Jimma Zone, South West Ethiopia: A cross-sectional study. Hindawi Publishing Corporation ISRN Public Health, Volume Article ID 238546, 7 pages. http://dx.doi. org/10.1155/2013/238546.

Astari, L.D., Nasoetion, A., \& Dwiriani, C.M. (2005). Hubungan karakteristik keluarga, pola pengasuhan dan kejadian stunting anak usia 6-12 bulan, (The relationship between family characteristics, parenting patterns and the incidence of stunting in children aged 
6-12 months). Media Gizi dan Keluarga, 29(2), 40-6.

Bappenas RI. (2012). Pedoman perencanaan program gerakan sadar gizi dalam rangka seribu hari pertama kehidupan (1000 HPK), (Guidelines for planning the nutrition awareness movement program for the first thousand days of life (1000 HPK)). p 1-8.

Fotso, J.C., Madise, N., Baschieri, A., Cleland, J., Zulu, A., ..., Mutua, A. et al. (2012). Child growt in urban deprived setting: Does household poverty status poverty status matter? At which stage of child development?. Health Place, 18(2), 375-384.

Giri, M.K.W., Suryani, N., \& Murdani K.P. (2013). Hubungan pengetahuan dan sikap ibu tentang pemberian ASI serta pemberian ASI eksklusif dengan status gizi balita usia 6-24 bulan (di Kelurahan Kampung Kajanan Kecamatan Buleleng), (The relationship between knowledge and attitudes of mothers about breastfeeding and exclusive breastfeeding with the nutritional status of children aged 6-24 months (in Kajanan Village, Buleleng District)). Jurnal Magister Kedokteran Keluarga, 1(1), 24-27.

Handayani, R. (2017). Faktor-faktor yang berhubungan dengan status gizi pada anak balita, (Factors related to nutritional status in children under five years old). Journal Endurance, 2(2), 217-224. DOI: http://doi. org/10.22216/jen.v2i2.1742.

Karima K., \& Achadi E.L. (2012). Nutrition status and infant birth weight. Jurnal Kesehatan Masyarakat Nasional, 7(3).

Kementerian Kesehatan Republik Indonesia. (2013). Naskah akademik pedoman gizi seimbang (PGS), (A balanced nutrition guideline (PGS) academic paper). Jakarta: Kementerian Kesehatan Republik Indonesia; p 1-27.

Kementerian Kesehatan Republik Indonesia. (2013). Rencana kerja pembinaan gizi masyarakat tahun 2013, (Community nutrition coaching work plan year 2013). Jakarta: Kementerian Kesehatan Republik
Indonesia.

Kementerian Kesehatan Republik Indonesia. (2013). Laporan hasil riset kesehatan dasar (Riskesdas) tahun 2013 [internet]: Status gizi anak balita, (Basic health research report (Riskesdas) 2013 [internet]: Nutritional status of children under five). Jakarta: Badan Penelitian dan Pengembangan Kesehatan; 2013. Diakses di http://www.depkes.go.id pada tanggal 3 Januari 2019 Pkl. 10.00 WIB.

Kementerian Kesehatan Republik Indonesia. (2017). Germas wujudkan Indonesia sehat. Kementerian Kesehatan Republik Indonesia, (Community movement to create a healthy Indonesia). Retrieved from http://www. depkes.go.id/article/view/16111500002/ germas-wujudkan-indonesia-sehat.html.

Khotimah, H., \& Kuswandi, K. (2014). Hubungan karakteristik ibu dengan status gizi balita di Desa Sumur Bandung Kecamatan Cikulur Regency Lebak tahun 2013, (The relationship between maternal characteristics and nutritional status of children under five in Sumur Village, Bandung, Cikulur District, Lebak Regency in 2013). Jurnal Obstretika Scientia, 2(1), 146-162.

Mohd Nasir, M.T., Norimah, A.K., Hazizi, A.S., Nurliyana, A.R., Loh, S.H., \& Suraya, I. (2012). Child feeding practices, food habits, anthropometric indicators and cognitive performance among preschoolers in Peninsular Malaysia. Appetite, 58(2), 525-530. https://doi.org/10.1016/j. appet.2012.01.007.

Nilakesuma, A., Jurnalis Y.D., \& Rusjdi, S.R. (2015). Hubungan status gizi bayi dengan pemberian ASI ekslusif, tingkat pendidikan ibu dan status ekonomi keluarga di wilayah kerja Puskesmas Padang Pasir, (The relationship between infant nutritional status and exclusive breastfeeding, mother's education level and family economic status in the working area of Puskesmas Padang Pasir). Jurnal Kesehatan Andalas, 4(1). ISSN: 2301-7406.

Ningsih, S., Kristiawati, \& Krisnana I. (2014). Hubungan perilaku ibu dengan status gizi 
kurang anak usia toddler, (The relationship between maternal behavior and malnutrition status of toddler). Jurnal Pediomaternal, $3(1), 58-65$.

Polit, D.F., \& Beck, C.T. (2008). Nursing research: Generating and assessing evidence for nursing practice, (8th Ed.). Philadelphia: Lippincott.

Riskesdas. (2013). Penyajian pokokpokok hasil riset kesehatan dasar 2013, (Presentation of the main results of basic health research 2013). Badan Penelitian dan Pengembangan Kesehatan, Kementerian Kesehatan RI. Akses www.litbang.depkes. go.id tanggal 10 Desember 2013.

Riskesdas. (2018). Hasil utama Riskesdas 2018, (Main results of Riskesdas 2018). Badan Penelitian dan Pengembangan Kesehatan, Kementerian Kesehatan RI.

Subarkah, T., Nursalam, \& Rachmawati, P.D. (2016). Pola pemberian makan terhadap peningkatan status gizi pada anak usia $1-3$ tahun (Feeding pattern toward the increasing of nutritional status in children aged $1-3$ years). Jurnal INJEC, 1(2), 146-154.

Suhardjo. (2002). Perencanaan pangan dan gizi, (Food and nutrition planning). Jakarta: PT Bumi Aksara.

Tan, L. (2011). Factor associated with exlusive breastfeeding among infants under six month of age in Peninsular Malaysia. Internasional Breastfeed Journal, 6(2).

United Nations Children's Fund (UNICEF). (2013). Improving child nutrition: The achievable imperative for global progress.

United Nations Children's Fund (UNICEF). Improving child nutrition, the achievable imperative for global progress. New York: United Nations Children's Fund.

United Nations Children's Fund (UNICEF). Ringkasan kajian gizi Oktober 2012, (Nutritional review summary in October 2012). Jakarta: UNICEF Indonesia.

USAID. (2014). Multi-sectoral nutrition strategy 2014-2025 technical guidance brief: Implementation guidance for ending preventable maternal and child death. P 1-6.

World Health Organization (WHO). (2010). Nutrition landscape information system: Country profile indicators. Geneva, Switzerland: World Health Organization. 